MRS Advances $\odot 2017$ Materials Research Society.This is an Open Access article, distributed under the terms of the Creative Commons Attribution licence (http://creativecommons.org/licenses/ by/4.0/), which permits unrestricted re-use, distribution, and reproduction in any medium, provided the original work is properly cited.

DOI: 10.1557/adv.2017.107

\title{
Additive Manufacturing, Modeling and Performance Evaluation of 3D Printed Fins for Surfboards
}

Reece D. Gately ${ }^{1}$, Stephen Beirne ${ }^{2}$, Geoff Latimer ${ }^{3}$, Matthew Shirlaw ${ }^{3}$, Buyung Kosasih ${ }^{4}$, Andrew Warren ${ }^{5}$, Julie R. Steele ${ }^{6}$, and Marc in het Panhuis ${ }^{1,2,3,7}$

${ }^{1}$ Soft Materials Group, School of Chemistry, University of Wollongong, Wollongong, NSW 2522, Australia

${ }^{2}$ Australian National Fabrication Facility Materials Node, University of Wollongong, Wollongong, NSW 2522, Australia

${ }^{3}$ Jones Beach Boardriders Inc., Kiama Downs, NSW 2533, Australia

${ }^{4}$ School of Mechanical, Materials and Mechatronic Engineering, University of Wollongong, Wollongong, NSW 2522, Australia

${ }^{5}$ School of Geography and Sustainable Communities, University of Wollongong, Wollongong, NSW 2522, Australia

${ }^{6}$ Biomechanics Research Laboratory, School of Medicine, University of Wollongong, NSW 2522, Australia

${ }^{7}$ ARC Centre of Excellence for Electromaterials Science, AIIM Facility, University of Wollongong, Wollongong, NSW 2522, Australia

\begin{abstract}
We demonstrate that Additive Manufacturing (3D printing) is a viable approach to rapidly prototype personalised fins for surfboards. Surfing is an iconic sport that is extremely popular in coastal regions around the world. We use computer aided design and 3D printing of a wide range of composite materials to print fins for surfboards, e.g. ABS, carbon fibre, fibre glass and amorphous thermoplastic poly(etherimide) resins. The mechanical characteristics of our 3D printed fins were found to be comparable to commercial fins. Computational fluid dynamics was employed to calculate longitudinal (drag) and tangential (turning) forces, which are important for surfboard maneuverability, stability and speed. A commercial tracking system was used to evaluate the performance of 3D printed fins under real-world conditions (i.e. surfing waves). These data showed that the surfing performance of surfboards with 3D printed fins is similar to that of surfboards with commercial fins.
\end{abstract}

\section{INTRODUCTION}

Surfing is an iconic sport that is extremely popular in coastal regions around the world. According to many reports Tom Blake (from USA) is generally given credit for inventing the surfboard fin in the 1930's [1]. Since then many different fin designs have been trialled, including famous high-aspect ratio fins designed by George Greenough in the 1960's [2]. These fins were wide at the base and narrower at the top resembling the dorsal fins of marine creatures such as dolphins. Fins glassed directly into the board were standard until the late 1960's, when removable fin systems became more widespread [1]. Today surfers can choose from a range of removable fin configurations including twin ( 2 fins), thruster ( 3 fins) and quad (4 fins) fins. Major manufacturers of removable fin systems include FCS and Futures [3, 4]. 
The manufacturing of commercial surfboard fins is currently based on injection molding. The mold making process is very expensive for one-off fin designs; hence, they are only used for running vast numbers of standard hydrofoil fins. Although this approach works for the masses, it does not offer potential for creating custom fins.

Additive manufacturing (AM), also known as 3D printing, describes a range of technologies that use computer aided design and automation to build structures through a layerby-layer process. Recently, it has seen a surge in activity and it is currently used in most research fields that depend on fabrication of materials, such as tissue engineering [5, 6]. Its use for fabrication of fins for surfboard is relatively new with a growing number of examples available on the internet [7]. However, most of these reports focus on the fabrication and modeling side of fin design and not on testing their performance in surfboards in the ocean.

In this paper, we used materials science and computational fluid dynamics (CFD) modeling to drive the development of Additive Manufacturing of 3D printed fins for surfboards [8]. The performance of surfboards with commercial and 3D printed fins was then measured using a commercial tracking system under real-world conditions, i.e. in a surfboard while surfing waves in the ocean.

\section{EXPERIMENT}

\section{$\underline{\text { Fin Design }}$}

The experimental fins were initially designed using computer aided design (CAD, Solidworks). A range of composite materials (ABS, carbon fibre composite and amorphous thermoplastic poly(etherimide) resins, (ULTEM)) and 3D printers (Markforged Mark2 and Dimension uPrintPlus, Objet Connex 350 and Fortus 900 instruments) were then used to fabricate the fins using $3 \mathrm{D}$ printing $[9,10]$. The fin designs were then modified based on the feedback provided by the recreational-level surfer, who quantitatively evaluated the fins in surfing trials conducted on waves in the ocean. This iterative cycle was repeated five times over several days of testing until the experimental fin design was established.

Commercial fins (e.g. FCS2 Performance Neoglass, FCS PC-5, FCS AM-1 green) were also purchased from local retail outlets (Wollongong area, Australia) as control fins [3]. The dimensions and material characteristics of the experimental and control fins are described in the Results and Discussion section.

The mechanical characteristics of experimental (3D printed) and control (commercial) fins were characterised with a custom-built setup consisting of a universal mechanical analyser (Shimadzu EZ-S) and a sample holder for clamping fins at the base (Figure 1a). This setup was used to evaluate the force required to flex the tip of the fins in the direction perpendicular to the fin using a circular probe (diameter $5 \mathrm{~mm}$ ) at a rate of $10 \mathrm{~mm} / \mathrm{min}$ using a circular tip (diameter 5 $\mathrm{mm}$ ). These data provided information about the flexibility of the fins and modulus (data not shown).

A computational fluid dynamics (CFD) analysis was carried out using Ansys CFX (2008 version). The CFD methodology was used to calculate the longitudinal and tangential turning forces of an idealized surfboard with fins in a thruster configuration. The incoming flow angle was varied from 0 to 15 degrees. 


\section{Fin Performance}

Fin performance was evaluated while two participants of varying surfing expertise performed standard surfing maneuvers on ocean waves. The first participant (recreational, intermediate level surfer; body mass $83 \mathrm{~kg}$, height 6'0", age 45 years) used a Dylan Perese (DP) Shadow surfboard (DP Surfboards, Thirroul, Australia; volume 30 L, length 5'11", width $191 \frac{1}{2}$ ") and a Dylan Longbottom Mexican surfboard (Dylan Shapes, Wollongong, Australia; volume 30.5 L, length 6'2", width 19 1/4") with either 3D printed ABS, carbon composites and ULTEM fins or commercial FCS2 Permormance or FCS GMB-5 fins in a quad configuration. The second participant (recreational, experienced level surfer; body mass $77 \mathrm{~kg}$, heigh 5'8", age 32 years) used a DP Rover surfboard (DP Surfboards, Thirroul, Australia; volume 26.75 L, length 5'10”, width $\left.187 / 8^{\prime \prime}\right)$ with either 3D printed ULTEM fins or commercial FCS Carver fins in a thruster configuration.

A commercial tracking system (TraceUp, USA), with 9 inertial sensors and a GPS was used to monitor and quantify the performance of the fins during each surfing trial. The tracker yielded angles (degrees; yaw, pitch, and roll, Figure $1 \mathrm{~b})$, linear and rotational speed $(\mathrm{m} / \mathrm{s}$ and $\mathrm{rad} / \mathrm{s}$; maximum and average), and power magnitude (a dimensionless number on a scale from 0 to 10 assigned by the tracking system) during characteristic surfing maneuvers (bottom turn and cutback, Figure 1c). TraceUp made the data of a professional surfer during bottom turns and cutbacks available to us. The dimensions of the surfboard, fins and the professional surfer were not disclosed.
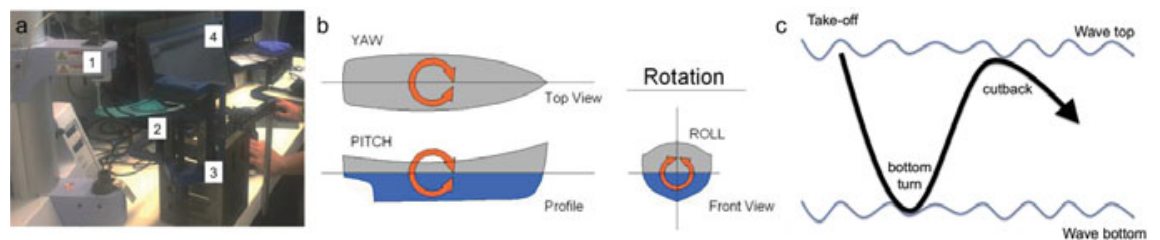

Figure 1. a) Photograph of the setup used to characterise the fins under laboratory conditions, numbers 1-4 indicate mechanical analyzer, fin, rig to clamp fin and computer for data collection, respectively. b) Schematic representation of the three rotational angles of a surfboard, yaw, pitch and roll. c) Schematic representation of the path of a surfer on a typical wave going from take-off via a bottom turn into a cutback.

\section{RESULTS AND DISCUSSION}

\section{$\underline{\text { Fin Design }}$}

The final specifications of the experimental fins used in the thruster configuration are detailed in Figure 2a. The comparatively smaller side fins of the quad fin arrangement were produced as a scaled version of the thruster fin design.

The mechanical properties of the experimental 3D printed and control (commercial) fins are shown in Figure 3. These data show that the mechanical characteristics (force-stroke curves) of the 3D fins printed in ULTEM and carbon fiber composites were found to be comparable to commercial FCS AM-1 fins. 


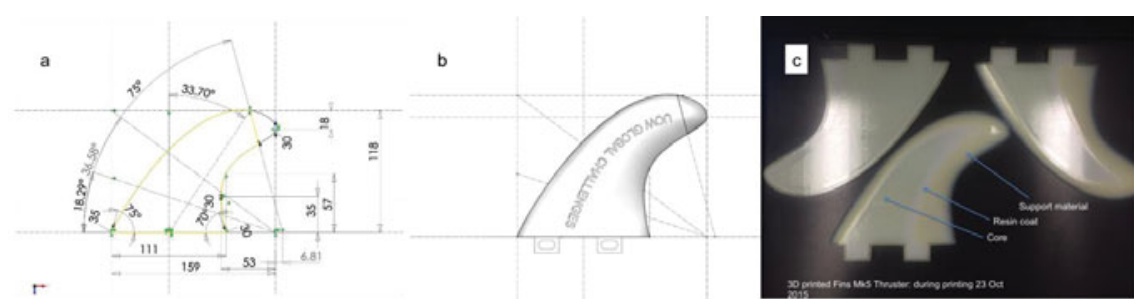

Figure 2. a) Screenshot of computer aided design (CAD) of fin. Numbers indicated parameters that can be varied in the design. b) CAD image showing lateral aspect of the fins c) Photo of typical 3D printed thruster fin set during printing on a Objet Connex printer.

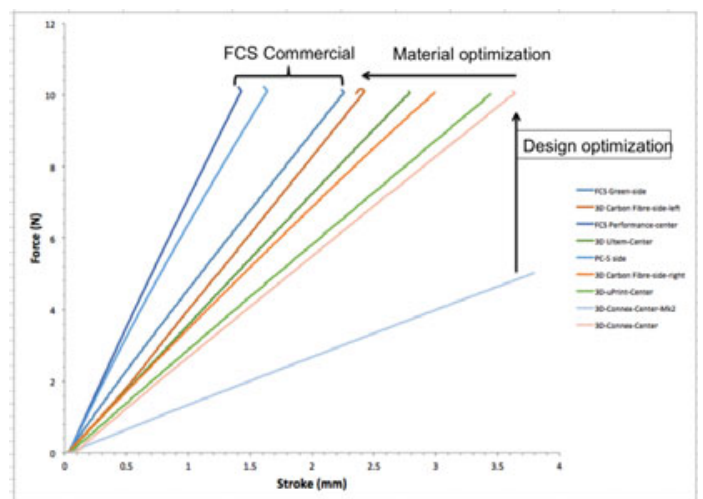

Figure 3. Applied force as a function of stroke of the experimental 3D printed and control surfboard fins tested under laboratory conditions. Vertical arrow, horizontal arrow and "FCS Commercial" indicate optimization of 3D printed fin designs, material optimization and commercial fins as manufactured by FCS.

Computational fluid dynamics (CFD) was used to calculate longitudinal (drag) and tangential (turning) forces, which are important for surfboard maneuverability, stability and speed. Figure 4 shows that incoming flow angles of more than 15 degrees can create swirls (vortices), which can produce substantial drag. It is suggested that incorporation of bumpy leading edges (Figure 4c) could reduce vortices and improve the efficiency of flow along the fins. Further research is necessary to validate this notion. 

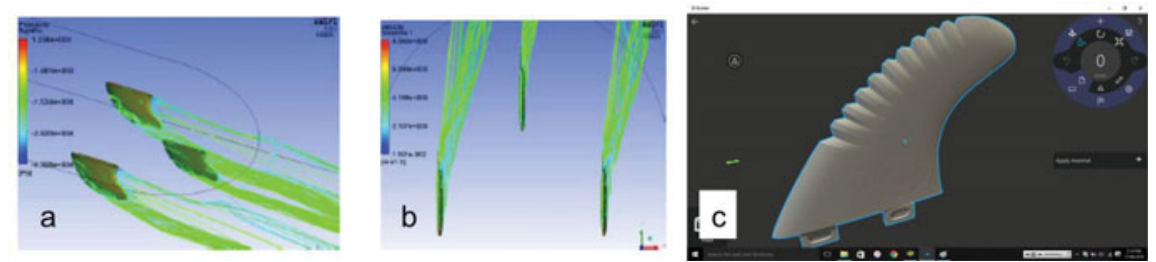

Figure 4. a) and b) Screenshots of computational fluid dynamics simulation of fins in an idealized surfboard under a yaw angle of 15 degrees. c) Screenshot of a computer aided design with bumpy leading edges on our fin design.

\section{Fin Performance}

The commercial tracking system used to monitor the performance of the experimental 3D printed and control (commercial) fins, while the participants performed bottom turns and cutbacks yielded a large amount of data, (see Figure 5 and Tables 1 and 2). A large sample size (number of waves surfed) was used to reduce the dependence on the input power/energy of the breaking waves.

3D fins printed in ULTEM and carbon fiber composites were found to be mechanically robust during surfing trials. In contrast, some of the $3 \mathrm{D}$ printed fins prepared using the $\mathrm{ABS}$ materials mechanically failed (at the base) during the trials. All data presented hereafter was generated using $3 \mathrm{D}$ fins printed in ULTEM and carbon fiber composites.
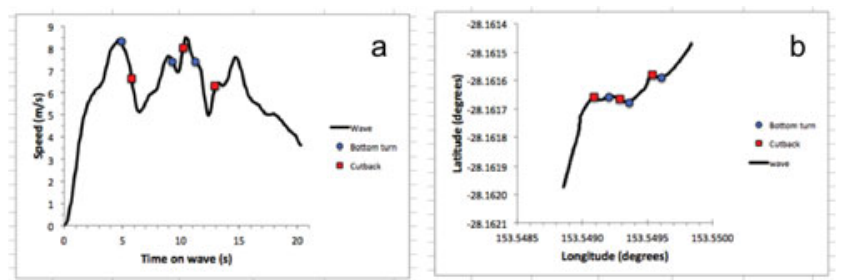

Figure 5. Examples data of Participant 2 riding a surfboard with 3D printed fins (ULTEM) in a thruster configuration on a typical wave (Snapper Rocks, Australia): a) Speed versus time. b) GPS data. Circles and squares indicate bottom turns and cutbacks, respectively.

Average linear and angular displacement and velocity data calculated for 1200 bottom turns and 1200 cutbacks performed by the two participants are summarized in Tables 1 and 2. In addition, we analysed the data for 512 bottom turns and 512 cutbacks performed by a professional surfer (see Table 2).

Surfing performance using the experimental (3D printed fins) was similar to that displayed when using the control (commercial) fins. For example, the average speed of the bottom turn performed by Participant 2 using the 3D printed fins $(7.2 \pm 0.9 \mathrm{~m} / \mathrm{s})$ and the commercial fins $(6.8 \pm 1.7 \mathrm{~m} / \mathrm{s})$ were highly consistent (Table 1$)$. In addition, the experimental (3D printed fins) were able to withstand cutback maneuvers performed with an average power magnitude of $5.2 \pm 2.4$, as generated by Participant 2 (note: power magnitude is a dimensionless number on a scale from 0 to 10 assigned by the tracking system). These cutback power 
magnitude values are similar to the values $(5.0 \pm 2.6)$ produced by Participant 2 when using commercial fins and are approaching the cutback power magnitude values $(5.8 \pm 3.2)$ that a professional surfer can generate when using commercial fins (Table 2).

Although speed decreases when surfers transition from a bottom turn into a cutback, this is coupled with an increase in rotational speed. In other words, the speed of the surfboard going into the bottom turn maneuver is used to increase the rotational movement of the surfboard to the benefit of the cutback maneuver. For example, Participant 2's (with 3D printed fins) speed decreased from $6.8 \pm 1.7 \mathrm{~m} / \mathrm{s}$ to $5.8 \pm 1.1 \mathrm{~m} / \mathrm{s}$ going from a bottom turn into a cutback.

Simultaneously, the rotational speed increased from $1.8 \pm 0.7 \mathrm{rad} / \mathrm{s}$ (bottom turn) to $2.4 \pm 0.6 \mathrm{~m} / \mathrm{s}$ (cutback). The values were highly consistent with the values generated by Participant 2 when using commercial fins (Tables 1 and 2).

We have quantified the power required to increase the rotational speed from bottom turn into cutback as a means to provide an additional measurement to compare the performance between surfers and the fins they use. This was deduced as follows. Power of a rotating body is equal to the product of torque and angular speed. Torque is the product of moment of inertia and angular acceleration (which in our case is the difference between the angular velocity of the bottom turn $\left(\omega_{\text {bott }}\right)$ and cutback $\left(\omega_{\text {cut }}\right)$ rotational speed divided by the duration). We assume that the moment of inertia of the surfboard can be approximated as a rectangular plane with a particular length (a) and width (b), where we assume that the weight (m), includes the weight of the surfer (as the plane is rotating with the surfer on it). This leads to the following equation for calculating the bottom turn to cutback power,

$$
\mathrm{P}=\mathrm{I} \alpha \omega_{\text {cut }},
$$

where, $\mathrm{I}$ is the moment of inertia calculated using $\left.\mathrm{m}\left(\mathrm{a}^{2}+\mathrm{b}^{2}\right) / 12\right)$, and $\alpha$ is the angular acceleration calculated using $\left(\omega_{\text {cut }}-\omega_{\text {bott }}\right) / t$. The resulting values are shown in Table 2 and clearly indicate that the experienced level surfer (Participant 2), generates/uses more power than the intermediate level surfer (Participant 1), i.e. $28.6 \pm 10.1 \mathrm{~W}$ versus $9.7 \pm 2.4 \mathrm{~W}$.

The data in Tables 1-2 clearly captures the technical ability of surfers of three different levels, intermediate, experienced and professional. For example, power, speed and angles generated are lowest for the intermediate level surfer and highest for the professional surfer. These data could be used to provide an indication of a surfer's skill-level either during free-surf or competition. The tracking data can also offer a complementary method to assess the scoring of waves during competitive surfing events, i.e. to assist the judges to award a score for a surfer's wave. Alternatively, scores could consist of two components, a judging score and a power score based on the data from the tracking system. However, the current tracking system is not equipped with live feedback of the data at present.

Table 1: Comparison of the summary data (generated using TraceUp commercial tracking system) for bottom turns carried out by Participants 1 and 2 using commercial and 3D printed fins. Values presented are the average ( \pm standard deviation) using a total sample size of 1200 bottom turns.

\begin{tabular}{|l|l|l|l|}
\hline & $\begin{array}{l}\text { Participant 2 } \\
\text { Commercial fins }\end{array}$ & $\begin{array}{l}\text { Participant 2 } \\
\text { 3D printed fins }\end{array}$ & $\begin{array}{l}\text { Participant 1 } \\
\text { 3D printed fins }\end{array}$ \\
\hline Speed $(\mathrm{m} / \mathrm{s})$ & $7.2 \pm 1.4$ & $6.8 \pm 1.7$ & $5.3 \pm 1.5$ \\
\hline Yaw angle $(\mathrm{rad})$ & $1.7 \pm 0.9$ & $1.5 \pm 0.7$ & $0.9 \pm 0.5$ \\
\hline Duration $(\mathrm{s})$ & $0.9 \pm 0.4$ & $0.8 \pm 0.4$ & $0.9 \pm 0.3$ \\
\hline Rotational speed $(\mathrm{rad} / \mathrm{s})$ & $1.9 \pm 0.9$ & $1.8 \pm 0.7$ & $1.0 \pm 0.5$ \\
\hline
\end{tabular}




\begin{tabular}{|l|l|l|l|}
\hline Roll angle (rad) & $0.8 \pm 0.2$ & $0.7 \pm 0.3$ & $0.6 \pm 0.4$ \\
\hline
\end{tabular}

Table 2: Comparison of the summary data (generated using TraceUp commercial tracking system) for cutbacks performed by Participants 1 and 2 using commercial and 3D printed fins. Summary data for professional surfer (calculated from the cutback data made available to us by TraceUp) is shown for comparison. Values presented are the average ( \pm standard deviation) using a total sample size of 1200 cutbacks (Participants 1 and 2) and 512 cutbacks (professional surfer). Power magnitude is a dimensionless number on a scale from 0 to 10 a dimensionless number on a scale from 0 to 10 assigned by the TraceUp tracking system.

\begin{tabular}{|l|l|l|l|l|}
\hline & $\begin{array}{l}\text { Participant 2 } \\
\text { Commercial } \\
\text { fins }\end{array}$ & $\begin{array}{l}\text { Participant 2 } \\
\text { 3D printed } \\
\text { fins }\end{array}$ & $\begin{array}{l}\text { Participant 1 } \\
\text { 3D printed } \\
\text { fins }\end{array}$ & $\begin{array}{l}\text { Professional } \\
\text { Surfer } \\
\text { Commercial fins }\end{array}$ \\
\hline $\begin{array}{l}\text { Power magnitude } \\
\text { (dimensionless) }\end{array}$ & $5.0 \pm 2.6$ & $5.2 \pm 2.4$ & $1.7 \pm 1.4$ & $5.8 \pm 3.2$ \\
\hline Speed (m/s) & $6.1 \pm 1.4$ & $5.8 \pm 1.1$ & $4.6 \pm 1.4$ & $6.8 \pm 2.3$ \\
\hline Yaw angle (rad) & $2.7 \pm 0.6$ & $2.6 \pm 0.6$ & $1.9 \pm 0.5$ & $3.0 \pm 0.3$ \\
\hline Duration (s) & $1.1 \pm 0.4$ & $1.1 \pm 0.3$ & $1.4 \pm 0.6$ & $1.1 \pm 0.1$ \\
\hline Rotational speed (rad/s) & $2.5 \pm 0.6$ & $2.4 \pm 0.6$ & $1.4 \pm 0.5$ & $2.7 \pm 0.5$ \\
\hline Roll angle (rad) & $1.1 \pm 0.4$ & $1.0 \pm 0.4$ & $0.9 \pm 0.5$ & $1.2 \pm 0.5$ \\
\hline Pitch angle (rad) & $0.8 \pm 0.3$ & $0.7 \pm 0.3$ & $0.6 \pm 0.4$ & $0.8 \pm 0.3$ \\
\hline $\begin{array}{l}\text { Bottom turn to Cutback } \\
\text { Power (W) }\end{array}$ & $29.8 \pm 11.0$ & $28.6 \pm 10.1$ & $9.7 \pm 2.4$ & - \\
\hline
\end{tabular}

\section{CONCLUSIONS}

In this paper, we have demonstrated that Additive Manufacturing (3D printing) is a viable approach to rapidly prototype fins for surfboards that can be personalised. The use of computer aided design and 3D printing utisiling a wide range of composite materials, e.g. ABS, carbon fibre, fibre glass and ULTEM allowed for the rapid design and fabrication of fins. The mechanical characteristics (under laboratory conditions) of the 3D printed fins were found to be comparable to commercial fins. A commercial tracking system used to evaluate the performance of 3D printed and commercial fins under real-world conditions, i.e. in a surfboard while surfing waves showed that the performance of a surfboard with $3 \mathrm{D}$ printed fins is similar to the performance of a surfboard using commercial fins.

This research has provided a pathway not only to fabricate fins for surfboards using 3D printing, but also to validate their performance in surfboards compared to commercial fins.

\section{ACKNOWLEDGMENTS}

The authors would like to thank the University of Wollongong Global Challenges program for financial support and the Australian National Fabrication Facility (ANFF) for providing access to facilities and expertise. D. Lokshin, C. Roberts-Thomson and V. Ishmuratov (all from TraceUp, USA), J. Witkovski (Emora Instruments, Australia) and Illawarra Sports High School (Berkeley, Australia) work experience students are thanked for technical 
assistance with accessing the Trace API, printing of carbon composite fins and mechanical testing, respectively.

\section{REFERENCES}

1. The Encyclopedia of Surfing by Matt Warshaw. Available at: http://encyclopediaofsurfing.com/ (accessed 16 December 2016).

2. B. Mctavish, Stoked! (Hyams Publishing, 2009) p. 27.

3. FCS Fin Systems. Available at: http://www.surffcs.com.au/fin-systems/fcs-ii (accessed 16 December 2016).

4. Futures Fin School. Available at: http://www.futuresfins.com.au/fin-school/ (accessed 16 December 2016).

5. H.-W. Kang, J. H. Park, T.-Y. Kang, Y.-J. Seol and D.-W. Cho, Biofabrication 4, 015005 (2012).

6. C.J. Ferris, K.G. Gilmore, G.G. Wallace, and M. in het Panhuis, Appl. Microbiol. Biotechnol. 97, 4243 (2013).

7. Westkust 3D printed fins. Available at: http://westkustsurf.nl/?page_id=19153 (accessed 16 December 2016).

8. 3D printed fins take surfing to the next level. Available at: https://youtu.be/96Zysk7WgVs (accessed 9 December 2016).

9. Carbon fiber composite. Available at: https://markforged.com/materials/\#carbon-fiber (accessed 16 December 2016).

10. ULTEM 9085 Resin, 3D print high performance parts. Available at: http://www.stratasys.com/materials/fdm/ultem-9085 (accessed 16 December 2016). 